\title{
Research on a New Type of Composite Islanding Detection Method
}

\author{
Wang Hao*, An Yuan, Wang Songkai, Ding Hang \\ Institute of Water Resources and Hydro-electric Engineering, Xi'an University of Technology, Xi'an, China
}

\section{Email address:}

1214699177@qq.com (Wang Hao), anyuan@xaut.edu.com (An Yuan)

${ }^{*}$ Corresponding author

\section{To cite this article:}

Wang Hao, An Yuan, Wang Songkai, Ding Hang. Research on a New Type of Composite Islanding Detection Method. Science Discovery. Vol. 5, No. 2, 2017, pp. 86-93. doi: 10.11648/j.sd.20170502.12

Received: March 24, 2017; Accepted: April 14, 2017; Published: April 20, 2017

\begin{abstract}
Distributed photovoltaic (PV) grid failure occurs, the short circuit fault current is small, and the power flow is bidirectional, the traditional power grid relay protection method is no longer applicable. In this article, through the study of traditional active frequency drift islanding detection method (AFD), analyses its advantages and disadvantages and applicable occasions, a new type of composite island detection method is proposed, the method based on adaptive islanding detection method accelerated frequency shift (AAFD), by introducing the common coupling point voltage frequency in a direction of continuous change number $n$, piecewise adaptively adjust frequency positive feedback coefficient, accelerate the limit of the frequency deviation, if the number of change if more than a dozen times, are not the limit of the frequency, the constant 2 cycle of negative pulse current amplitude power frequency interference, trigger under-voltage protection, so as to detect the island.this method can reduce the grid harmonic distortion rate, improve the response time of the island detection. At last, through Matlab simulation to verify.
\end{abstract}

Keywords: Distributed Photovoltaic, An Island Detection, Frequency Deviation, The Adaptive

\section{一种新型复合孤岛检测方法的研究}

王浩*, 安源, 王颂凯, 丁航

水利水电学院, 西安理工大学, 西安, 中国

邮箱

1214699177@qq. com（王浩）, anyuan@xaut.edu.com（安源）

摘要：分布式光伏并网发生故障时, 短路故障电流小, 潮流双向流动, 传统的大电网继电保护方法已不再适用。本文 通过研究传统的主动频率偏移孤岛检测方法 (AFD), 分析了其优缺点及适用场合, 提出了一种新型复合孤岛检测方法, 该方法基于自适应加速频移的孤岛检测方法（AAFD），通过引入了公共耦合点电压频率朝某个方向的持续变化次数 $\mathrm{n}$, 自适应地分段调节频率正反馈系数, 加速频率偏移越限, 若该变化次数如果超过 12 次, 频率仍未越限, 则施加持续 2 个工频周期的负脉冲电流幅值干扰，触发欠压保护，从而检测出孤岛。该方法可以减小并网谐波畸变率，提高孤岛检 测的响应时间。最后通过Matlab仿真得以验证。

关键词: 分布式光伏, 孤岛检测, 频率偏移, 自适应 


\section{1. 引言}

随着经济的发展, 电力用户对用电可靠性、电能质量 的要求越来越高。传统集中式发电面临严格限制, 不能在 可接受的成本内满足敏感电力负荷需求。与此同时，技术 进步使得分布式发电重新回到电力发展的舞台。分布式发 电具有灵活、高效和环保优势, 可以提供更加清洁, 优质 的电能, 且节省了电力输送所需的大量投资, 经济性比较 显著。随着分布式发电逐渐大规模接入配电网, 给配电网 也带来了很多问题, 其中孤岛效应问题是最值得探讨的问 题之一。
孤岛效应是指当大电网因故障、停电检修或人为因素 等原因而跳闸断电时, 各分布式电源未能检测出断电状态, 继续向周围负荷供电, 最终形成自给供电孤岛的现象 [1]。 反孤岛保护就是当探测到光伏电站处于孤岛运行状态时, 能够断开光伏电站与孤岛系统的连接, 破坏孤岛运行条件 [2] [3]。通过检测公共耦合点 (Point of Common Coupling, PCC) 的频率或电压是否超过国家标准来判断是否封锁并 网逆变器。如图1所示, 电网正常时 (断路器闭合), 由 分布式光伏系统与电网共同给负载供电; 电网断开后 (断 路器断开), 仅由光伏系统给负载供电, $\mathrm{a}$ 点即为公共耦 合点（PCC）。

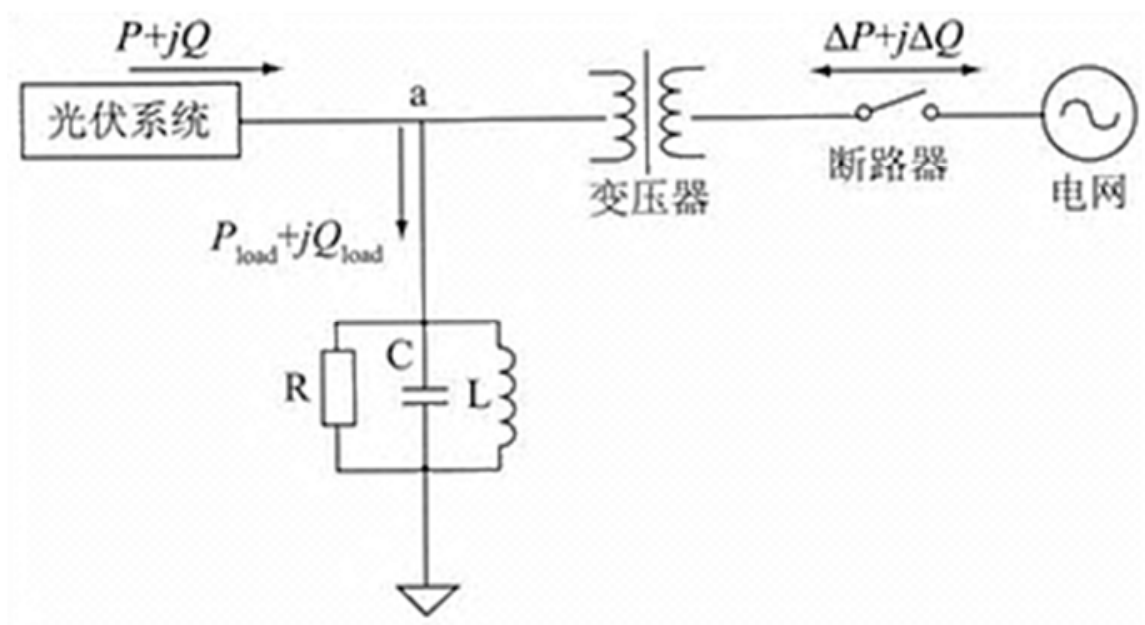

图1 光伏并网示意图。

一旦孤岛效应发生, 将会使系统电压频率和幅值不稳 定, 引起电网重合闸, 造成工作人员触电等严重的后果。 因此, 对于含有分布式光伏的系统而言, 必须具备孤岛检 测的功能, 即必须具有检测孤岛效应和及时与电网断开的 能力。

孤岛检测的方法一般分为被动检测法和主动检测法。 被动检测法 (也称无源检测) 是根据直接测量得到的电压 和电流信号, 来判断相位、频率和幅值是否超过检测阈值 (即国家标准) [4]。常用的被动检测法有电压过高/过低、 频率过高/过低检测法, 电压相位突变检测法 (PJD), 电 压谐波和电流谐波检测法（DVH、DCH）。主动检测法（也 称有源检测) 是指在并网逆变器的指令控制信号中, 施加 很小的频率、相位或电压扰动信号, 检测并网侧相位、频 率和幅值是否超过检测阈值 [5]。主动检测法主要分为两 类, 频率法和幅值法。频率类的检测方法主要有主动频率 偏移法 (AFD) 、带正反馈的主动频率偏移法 (AFDPF)、2N 电压周期电流扰动法、频率正反馈的孤岛检测方法、滑膜 频率偏移法 (SMS) 等; 幅值类的检测方法主要有电压前馈 正反馈扰动检测方法、电流前馈正反馈扰动检测方法、周 期交替电流扰动法、主动电流扰动法、无功补偿法等。被 动检测法的缺点是存在很大的检测盲区 (Non Detection Zone，NDZ），一般配合主动检测法一起应用 [6] [7]。现 在国内外应用最多的是主动频率偏移法 (Active Frequency Drift, AFD）。文献 [8]在分析传统AFD法基础 上, 提出了一种施加脉冲电流幅值干扰的改进方法, 并且
讨论了电流幅值干扰施加的方式和施加的时间。文献 [9] 中采用直接加入相角的方式来移相, 是为了克服传统AFD 中用截断电流方式移相而导致的谐波问题。文献 [10]通过 对最优检测点间隔进行估计和频率奇异点的剔除, 提出了 抗干扰六点测频法, 该方法可有效避免因电网干扰对主动 频移法产生不利影响而导致的孤岛效应误判现象, 提高了 孤岛检测的可靠性。但是, 上述文献所使用的方法, 难以 同时兼顾孤岛检测的快速性和低谐波畸变率。

本文在传统的AFD法的基础上, 引入了PCC点电压频率 朝某个方向的持续变化次数 $\mathrm{n}$, 可以自适应地分段调节频 率偏移正反馈系数 $K$, 加速频率偏移越限, 且若该变化次 数如果超过 12 次, 频率仍未越限, 则施加持续 2 个工频周 期的负脉冲电流幅值干扰, 触发欠压保护, 从而检测出孤 岛。最后, 通过Matlab仿真进行了验证。

\section{2. 主动频率偏移法}

\section{1. 传统的主动频率偏移法（AFD）}

主动频率偏移法 (Active Frequency Drift, AFD) 的原理是: 控制光伏并网扰动电流的频率, 使光伏并网逆 变器输出电流频率比PCC点电压的频率略高或略低, 当电 网供电正常时, PCC点频率受电网频率的钳制不变, 当电 网断开时, PCC点频率将受给定扰动电流频率的影响发生 
偏移, 最终超出设定的阈值而封锁逆变器, 实现孤岛保护 [13]。

传统AFD法对逆变器输出的并网电流注入一定的畸变, 如图2所示。定义截断系数 $\mathrm{c} f$ 为电压过零点超前 (或滞后) 电流过零点的时间 $\mathrm{t}_{\mathrm{z}}$ 与电压周期的一半 $\mathrm{T} / 2$ 的比值, 见式 (3) 。其中V为公共耦合点电压, $i$ 为逆变器输出电流波 形, $i$ 与 $V$ 的相位差总是固定不变的, 即截断系数 $\mathrm{c} f$ 是不变 的。在每个周期的上升沿过零点时, 给并网电流加一个频 率的扰动。当电网电压正常时, 由于电网的容量可视为无
穷大, PCC点的电压被钳制为电网电压, 加入的频率扰动 对PCC点电压没有影响。当电网断路器断开时, PCC 点电压 由流过负载的电流和负载共同决定。而流过负载的电流的 频率由上个周期负载电压的频率决定，即本周期负载电流 的频率等于上周期检测到PCC点电压频率与频率扰动相加。 此电流加到负载上后, 会使负载电压的频率也发生变化。 经过几个周期的积累, 负载电压的频率会不断偏移, 直到 超出之前设定的阈值, 可判断系统发生孤岛。

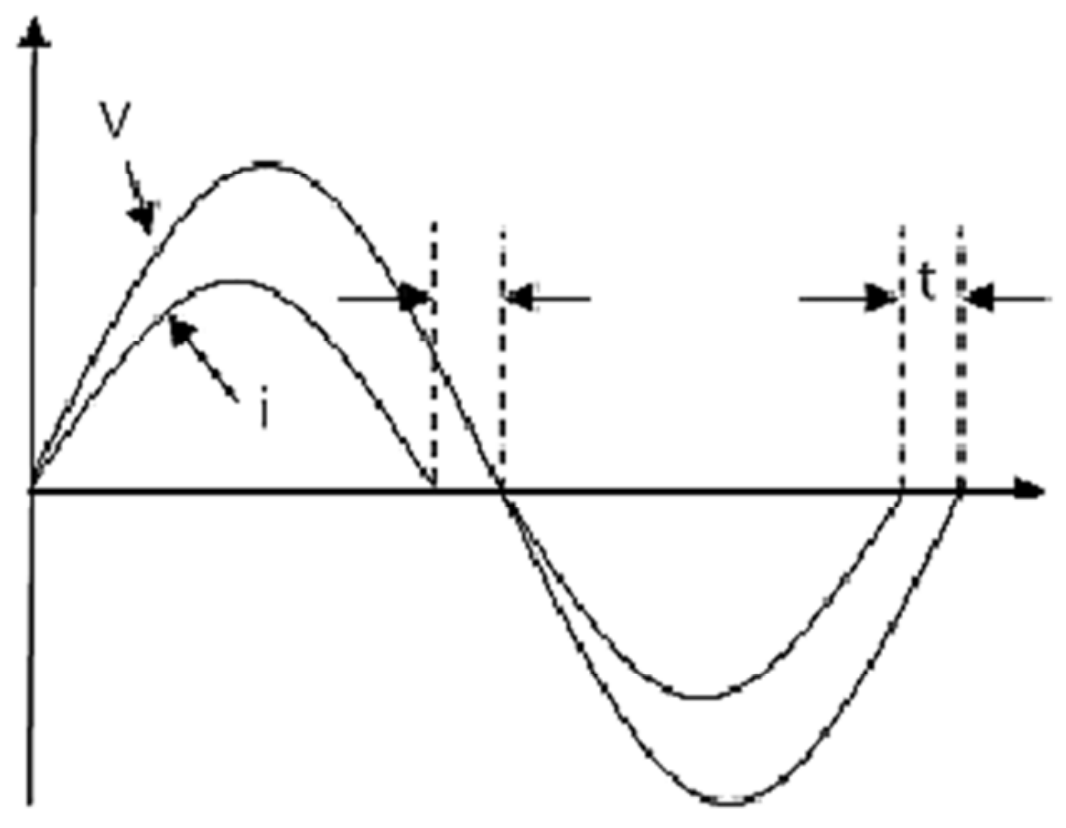

图2 主动频移法孤岛检测示意图。

检测PCC点电压的频率, 依其而添加并网扰动电流 $i_{r e f}$ 的频率。并且, 若 $i_{r e f}$ 半波己完成而 $\mathrm{U}_{0}$ 未到过零点, 则 $i_{r e f}$ 被 强制为零, 直到 $\mathrm{U}_{0}$ 过零触发到来, 也就是说 $i_{r e f}$ 按半波发 出, 起点是 $\mathrm{U}_{0}$ 的过零点, 其表达式如式 (1):

$$
i_{\text {ref }}=\left\{\begin{array}{lr}
-I_{0} \sin \frac{2 \pi}{T_{i}}\left(t-\frac{T_{u}}{2}\right), & T_{u} \leq t \leq T_{u}-t_{z} \\
I_{0} \sin \frac{2 \pi}{T_{i}} t, & 0 \leq t \leq \frac{T_{u}}{2}-t_{z}
\end{array}\right.
$$

式中, $I_{0}$ 一并网扰动电流幅值;

$T_{i}$ 一并网扰动电流周期;

$T_{u}$ 一公共耦合点电压周期;

$t_{z}$ - AFD的截断时间。

其中

$$
T_{i}=T_{u}-2 t_{z}
$$

定义截断系数 $\mathrm{c} f$ 为

$$
c f=\frac{2 t_{z}}{T_{u}}
$$

AFD容易实现, 效率较高, 相对于被动检测法, 检测 盲区减小, 多台并网时不需要通信, 但由于引入电流扰动, 对电能质量有一定影响。由于逆变器输出电流的扰动信号
都是按单一方向加入的, 所以电网断开后, 当负载特性不 同时, 逆变器输出电流的频率变化方向可能与频率扰动的 方向相反, 这样会使逆变器输出电压的频率变化积累较慢, 延长了孤岛的检测时间。当负载的阻抗角与加入扰动信号 所产生的角度相匹配时, 逆变器输出的电压频率将不会发 生变化, 产生检测盲区。

\section{2. 引入正反馈的主动频率偏移法 (AFDPF)}

在AFD法的基础上提出的带正反馈的主动频率偏移法 [16] [17], 其基本思想是在公共点电压波动时引入正反馈 加大电压与电流的相位差从而加速波动。此时, 截断系数 $\mathrm{c} f$ 是一直变化的, 其式如下:

$$
\mathrm{c} f=c f_{0}+k\left(f_{u}-f_{g}\right)
$$

式中, $c f_{0}$ 一初始时的截断系数;

$k$ 一反馈系数;

$f_{u}$ 一公共点电压的频率;

$f_{g}$ 一电网电压的额定频率, 取 $50 \mathrm{~Hz}$ 。

由于截断系数 $\mathrm{c} f$ 过大会导致光伏并网逆变器输出的 电流总谐波畸变率 (TotalHarmonicDistortion, THD) 值增 大，因此 $c f_{0}$ 可取为 0 ，因此得到式 (5)： 


$$
\mathrm{c} f=k\left(f_{u}-f_{g}\right)
$$

为反映品质因数 $Q_{f 0}$ 与孤岛检测的关系, 国内学者采 用 $Q_{f 0} \times C_{n o r m}$ 坐标平面对检测盲区进行描述, 参数定义如 下:

$$
\begin{gathered}
\mathrm{Q}_{\mathrm{f} 0}=\frac{\mathrm{R}}{\omega_{0} \mathrm{~L}} \\
\mathrm{C}_{\text {norm }}=\frac{\mathrm{C}}{\mathrm{C}_{\text {res }}} \\
\mathrm{C}_{\text {res }}=\frac{1}{\omega_{0}^{2} \mathrm{~L}} \\
\mathrm{C}=\left(1+\Delta \mathrm{C}_{\text {norm }}\right) \mathrm{C}_{\text {res }} \\
\mathrm{C}_{\text {norm }}=1+\Delta \mathrm{C}_{\text {norm }}
\end{gathered}
$$

文献 [17]提出了孤岛检测失败条件的相角判据, 文献 [18]在 [17] 基础上结合式 (6)-(10) 可得到式(11)：

$$
\Delta \mathrm{C}_{\mathrm{norm}}=\frac{\tan \left(\frac{\pi}{2} c f\right)}{Q_{f 0}}-\frac{2 \Delta f}{f_{0}}
$$

频率偏移 $\Delta \mathrm{f}$ 允许的范围为 $[-0.5,0.5]$, 由式 (5)、 式 (11) 和式 (12) 可得到正反馈的主动频率偏移法法的 检测盲区为:

$\frac{\tan \left(\frac{\pi}{2} k \times 0.5\right)}{Q_{f 0}}-\frac{0.5}{f_{0}}+1<C_{n o r m}<\frac{\tan \left(-\frac{\pi}{2} k \times 0.5\right)}{Q_{f 0}}+\frac{0.5}{f_{0}}+1$

式中 $Q_{f 0}$ 一品质因数;

$C_{\text {norm }}$ 一标准化电容。

由式 (12) 可得到如图2所示的孤岛检测盲区图。

与AFD相比, 在相同的相位差下, AFDPF能有效减小检 测盲区, 加快了检测速度, 缓解了输出电能质量与检测效 率的矛盾, 多台并网时也不需要通信。

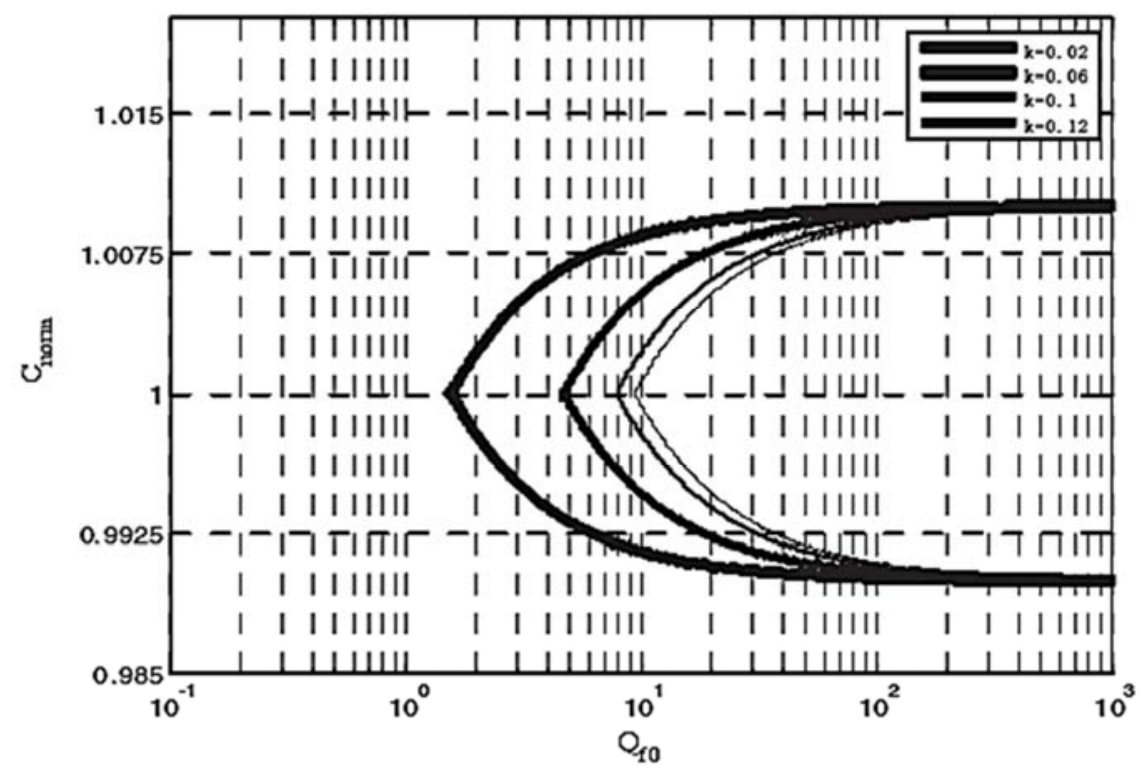

图3 检测盲区图。

由图 3 可见: 反馈系数 $k$ 越大, 盲区就越小。因此, 只 要反馈系数 $\mathrm{k}$ 取值足够大, 完全可以消除盲区。但是, 正 反馈系数k越大, 电流畸变率越大, 对电能质量产生影响 则越大。因此, 对于孤岛检测来说, 无盲区NDZ和电流畸 变率THD两者相互矛盾。而目前大量文献中, $\mathrm{k}$ 值为固定值, 很难在NDZ和THD间达到很好的平衡。因此对于正反馈系数 $k$ 值的选取, 成为了本文研究的重点。

\section{3. 自适应加速主动频率偏移法 (AAFD)}

为了更好地兼顾NDZ和THD, 本文提出动态自适应选取 正反馈系数 $\mathrm{k}$ 值的思路, 具体做法为引入PCC处电压频率朝 某个方向的持续变化次数 $\mathrm{n}$, 从而根据 $\mathrm{n}$ 的具体取值而自适 应的按分段函数 $\mathrm{f}(\mathrm{n})$ 选取相应的正反馈系数 $\mathrm{k}$, 如式 (13) 所示, 若PCC处电压频率朝着增加的方向持续变化1次, 则 $\mathrm{n}$ 自增 1 ; 若朝着减小的方向持续变化 1 次, 则 $\mathrm{n}$ 自减 1 。

$$
\mathrm{k}=\mathrm{f}(\mathrm{n})=\left\{\begin{array}{lr}
0.01, & |n| \leq 3 \\
0.015(|n|-3), & 3<|n| \leq 12 \\
0, & |n|>12
\end{array}\right.
$$

由式 (4)、（13）可得

$$
\mathrm{cf}=\left\{\begin{array}{lr}
0.01\left(\mathrm{f}_{\mathrm{u}}-50\right), & |\mathrm{n}| \leq 3 \\
0.015(|\mathrm{n}|-3)\left(\mathrm{f}_{\mathrm{u}}-50\right), & 3<|\mathrm{n}| \leq 12 \\
0, & |\mathrm{n}|>12
\end{array}\right.
$$

根据GB/T15945-1995规定, 电力系统正常频率偏差 允许值为 $0.2 \mathrm{~Hz}$ 。考虑波动最大达到 $0.2 \mathrm{~Hz}$ 的情况, 由式 (14) 可见, 只要 $|n|$ 不超过 3 次, 则 $\mathrm{f}=0.001$, 此时施加的干扰 为低畸变率的频率。当 $|n|$ 超过 3 次时, 加强频率干扰, 且 截断系数 $\mathrm{cf}$ 随 $|n|$ 的增加而增加, 孤岛检测的盲区逐渐缩小, 加速了频率越限, 从而检测出孤岛; 当 $|n|$ 超过 12 次时, 可 
以肯定电网发生了故障, 则 $\mathrm{cf}=0$, 此时施加负脉冲电流幅 值干扰。

根据式 (1) 和式 (14) 用Matlab/Simulink构建一可编 程电流源作为并网扰动电流, 频率波动则考虑电网频率波 动达到 $0.2 \mathrm{~Hz}$ 的最坏情况, 改变 $n$ 值可得到并网扰动电流波
形, 检测其THD, 可以更加清楚地说明 $\mathrm{n}$ 与 THD之间的关系, 如此通过若干组数据描点得到图 4, 由图可见: THD的取值 不超过 $2.8 \%$ 。而IEEEStd929-2000规定, 注入电网电流的 总谐波畸变率THD不能大于 $5 \%$, 因此完全满足标准。

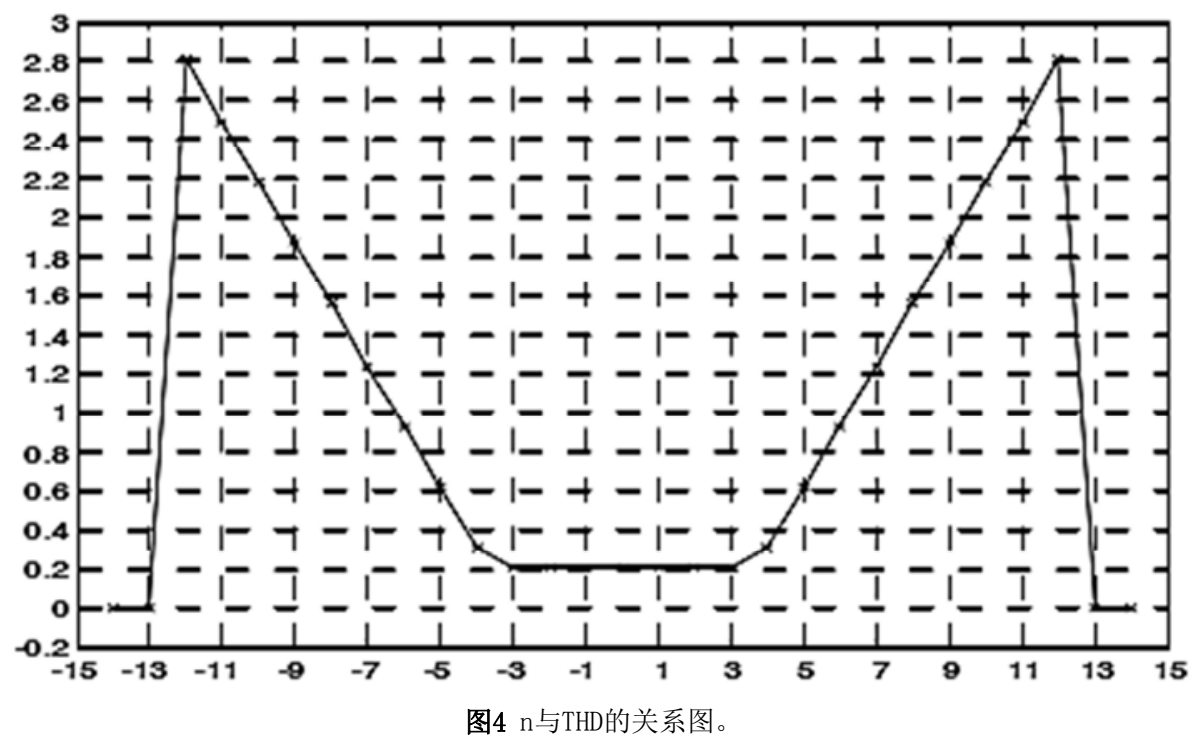

现在大多数文献中电流幅值干扰法是周期性的对电 流幅值施加脉冲扰动 [19] [20], 这种方法会延迟孤岛检测 的时间。因此, 本文提出负脉冲电流幅值干扰法: 当 $|n|$ 超 过 12 次时, 说明在主动频率干扰的作用下, PCC处电压频 率已朝某个方向持续变化了至少 12 次, 可以断定此时已发
生故障, 为保证在自动重合闸时间 $0.3 \mathrm{~s}$ 内快速准确地检测 出孤岛, 此时直接施加负脉冲干扰, 且负脉冲持续 2 个工 频周期。 5所示.

综上, 本文所提出的复合型自适应孤岛检测方法如图

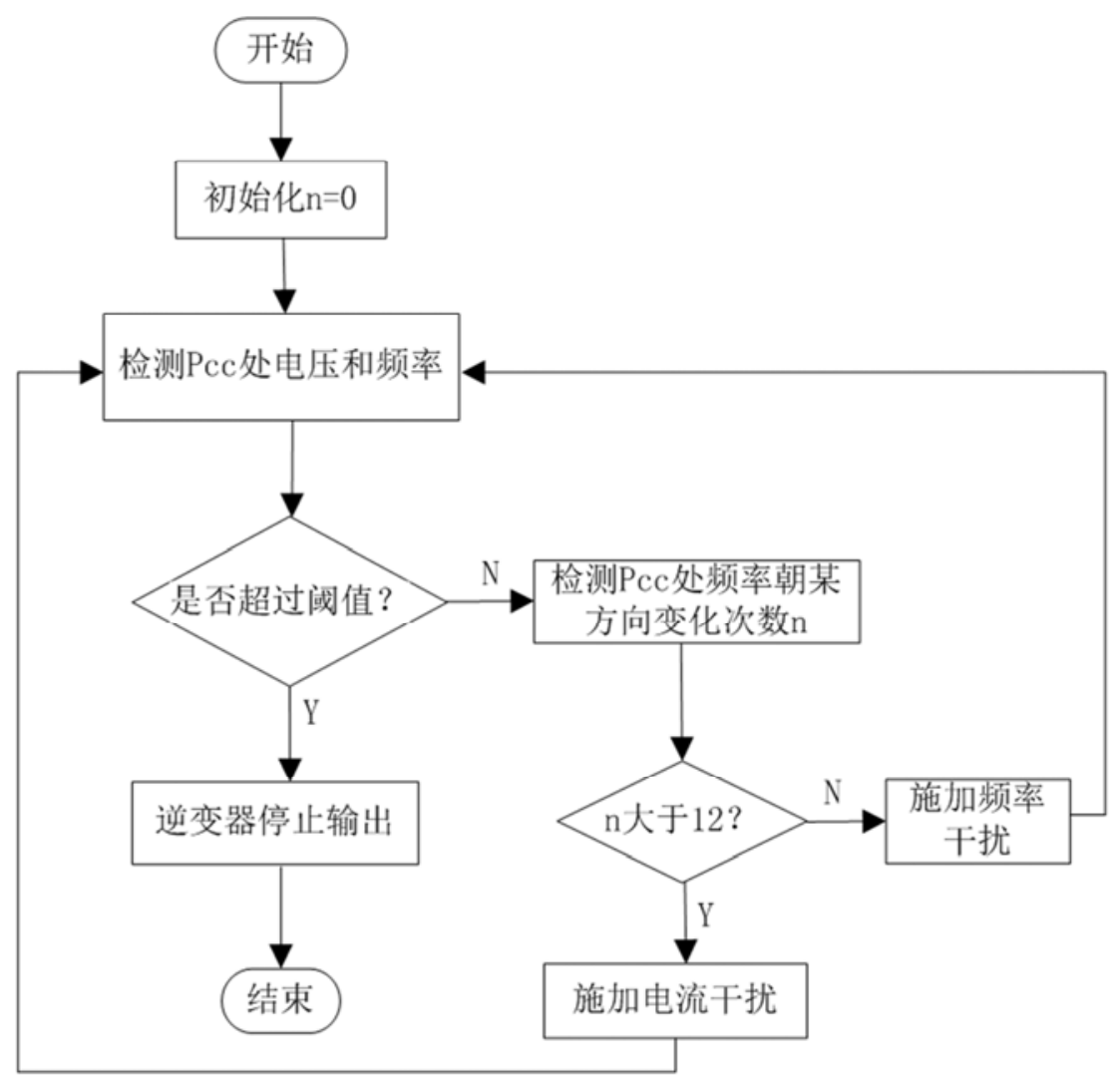

图5 复合孤岛检测方法流程图。 


\section{3. 仿真验证}

针对光伏发电系统的孤岛检测, 通过 Matlab/Simulink进行了仿真, 逆变器采用电流控制模式, 负载采用与逆变器输出功率相平衡的RLC并联负载 (品质
因数 $\mathrm{Q}_{\mathrm{r}}=2.5, \mathrm{R}=6.2252, \mathrm{~L}=7.9 \mathrm{mH}, \mathrm{C}=1300 \mu \mathrm{F}$ ) 。在 $0.3 \mathrm{~s}$ 时, 断开断路器, 要求孤岛检测算法必须在高速自动重合闸时 间 (即 $0.3 \mathrm{~s}$ ) 内检测出孤岛, 封锁逆变器, 实现有效的孤 岛保护。仿真结果如图6一图12所示:

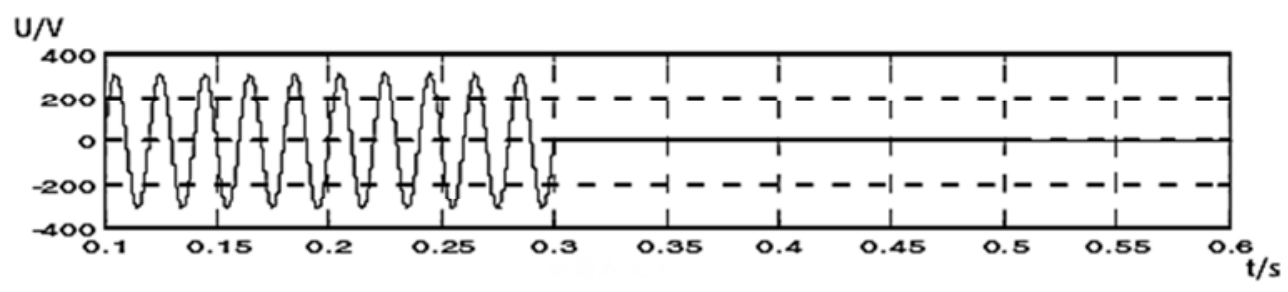

图6 电网电压。

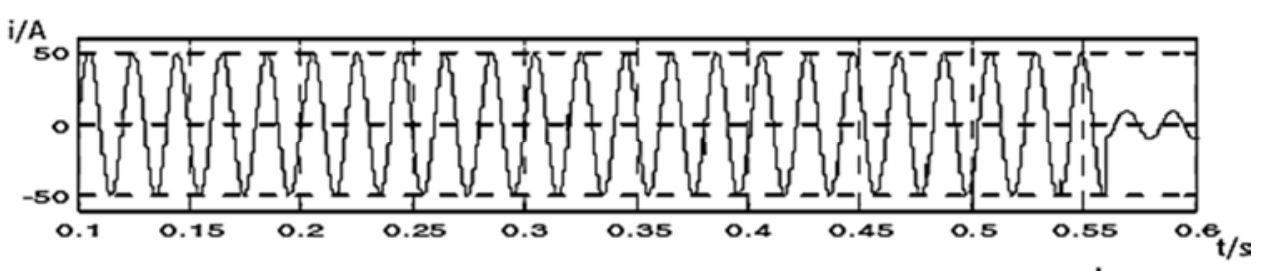

图7 逆变器输出电流。

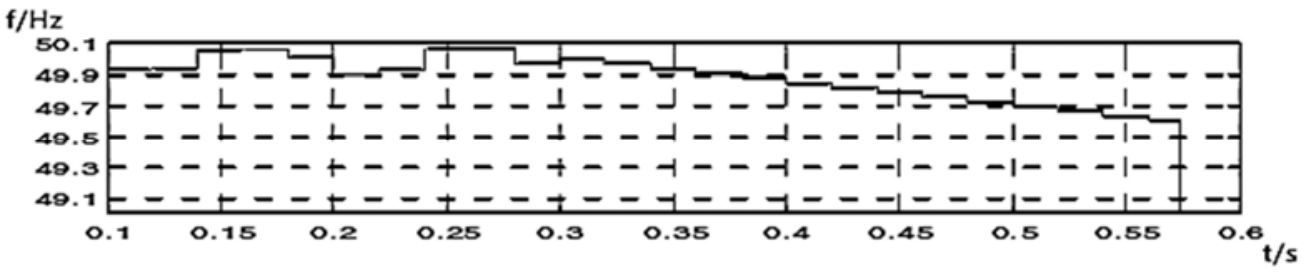

图8 PCC点电压频率。

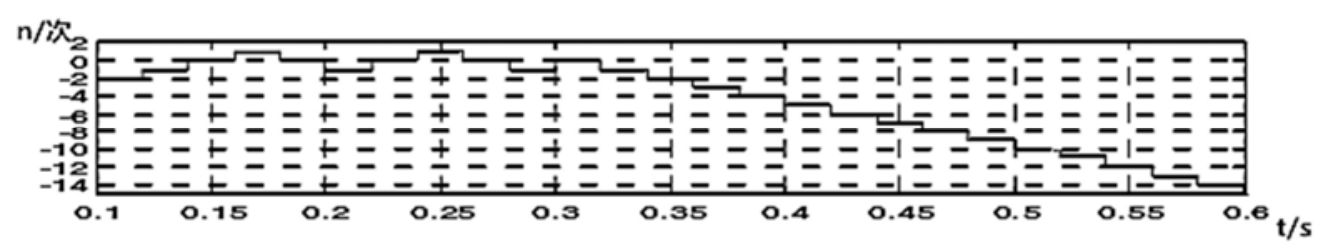

图9 频率朝某方向变化次数。

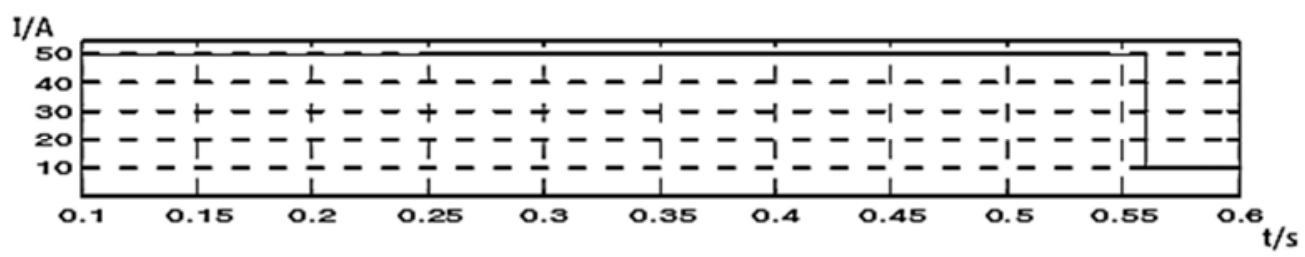

图10 逆变器的输出电流幅值。

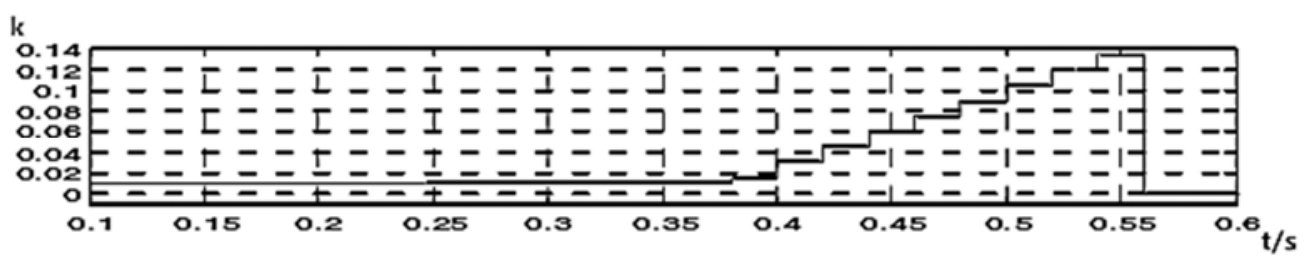

图11 正反馈系数K。 


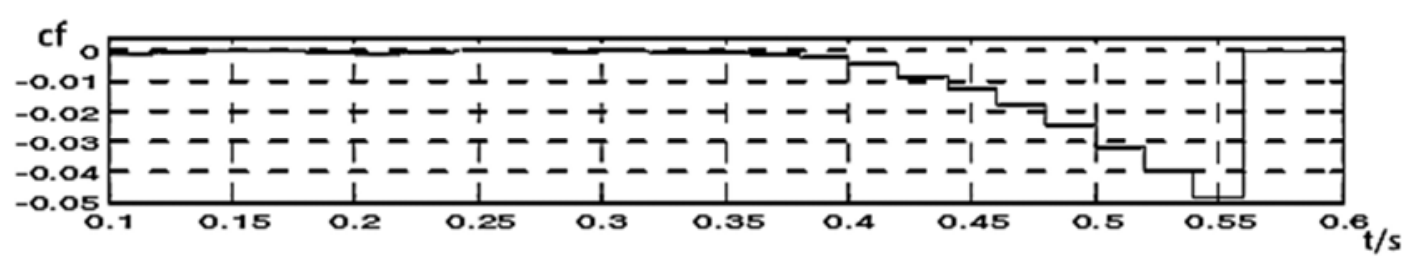

图12 截断系数cf。

在开始的 $0.1 \mathrm{~s}-0.3 \mathrm{~s}$ 中, 光伏系统与电网并联运行, 电网电压为稳定的正弦波 (如图6)。且检测到的电网频 率在 $50 \pm 0.2 \mathrm{~Hz}$ 范围内波动（如图8）。由于 $|n|$ 没有超过 3 次, 认为没有孤岛现象发生, 正反馈系数为 0.01 (如图 11 )。 截断系数 $\mathrm{cf}$ 不超过 0.001 (如图12）。此时施加低畸变率 频率干扰, 光伏逆变器输出电流总谐波畸变率仅为 $0.56 \%$ （如图13）。

在 $0.3 \mathrm{~s}$ 时电网断电, $|n|$ 超过 3 次后, 正反馈系数 $\mathrm{k}$ 以 0.015 为斜率, 随 $|n|$ 的增加而成比例的增加（如图11）,
相应地截断系数 $\mathrm{cf}$ 也逐渐朝负方向增大 (如图12), 加速 PCC点电压频率向欠频保护值49.5 Hz逼近（如图13）。

当 $|n|=12$ 时, 如图8所示, 对应的时间为 $0.56 \mathrm{~s}$, 由图 9 可知, 此时频率为 $49.64 \mathrm{~Hz}$, 没有达到欠频保护值 $49.5 \mathrm{~Hz}$, 由于 $\mathrm{u}_{0}$ 小于欠压保护值而触发欠压保护, 逆变器在 $0.575 \mathrm{~s}$ 时封锁PWM脉冲输出, 整个实现孤岛保护的时间仅为 $0.275 \mathrm{~s}$, 成功实现在 $0.6 \mathrm{~s}$, 即高速自动重合闸动作之前停 止逆变器的并网运行, 且施加负脉冲电流幅值干扰前逆变 器输出电流的总谐波畸变率仅为 $4.52 \%$ （如图14）, 低于 国家谐波标准 $5 \%$ 。

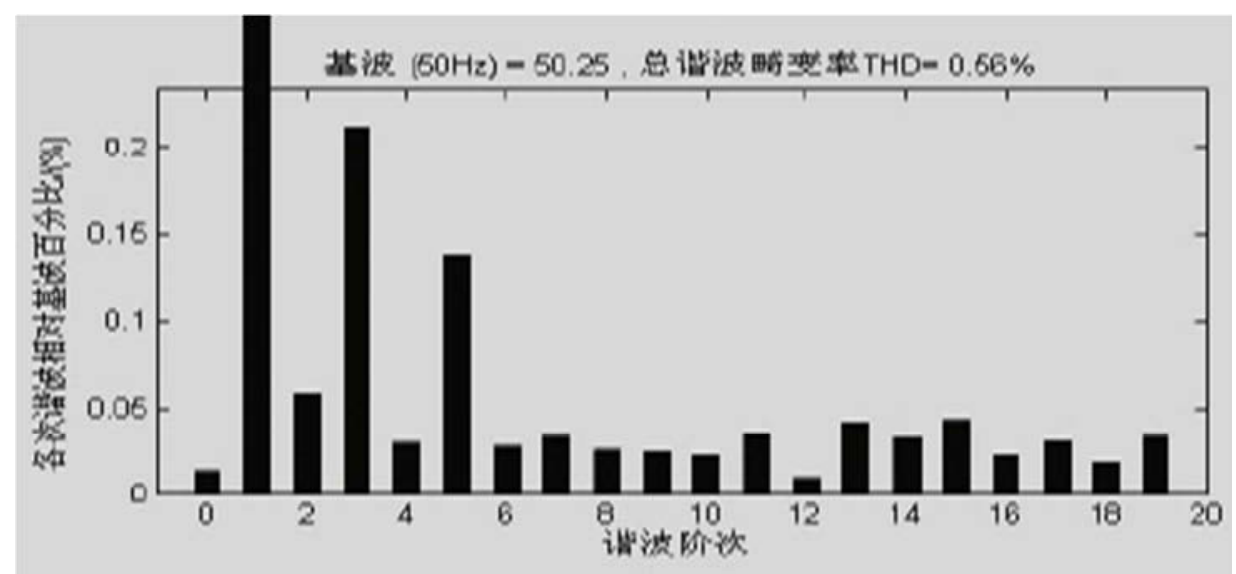

图13 $|\mathrm{n}| \leqslant 3$ 时的电流谐波畸变率。

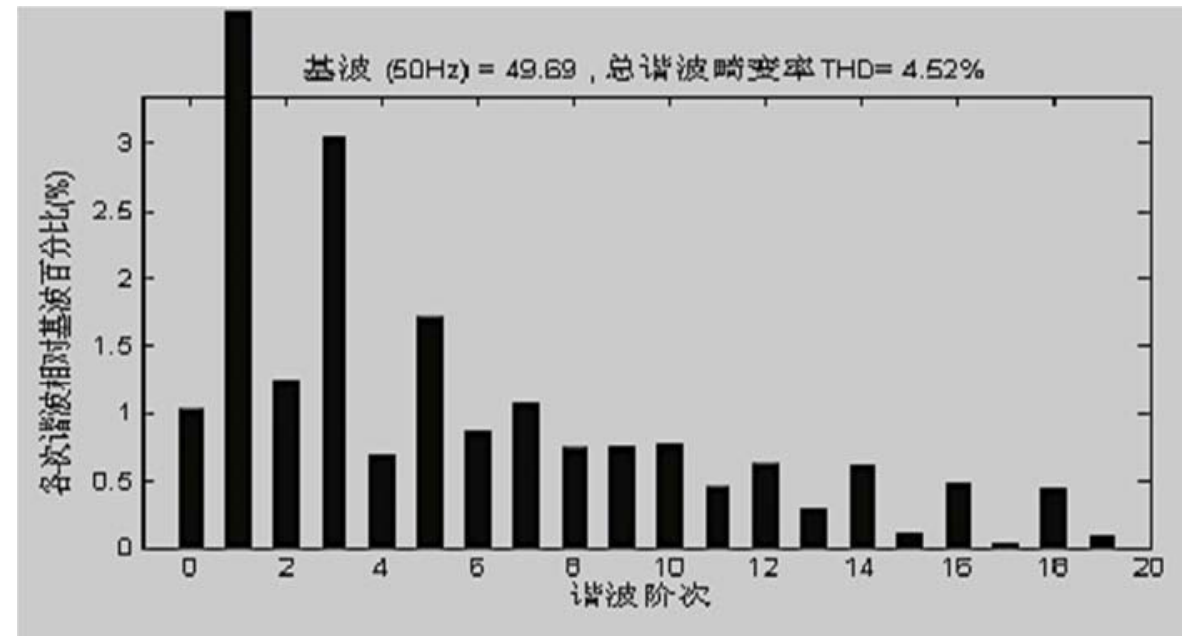

图14 $3<|\mathrm{n}| \leqslant 12$ 时电流谐波畸变率。

\section{4. 结论}

本文在传统的主动频移检测方法的基础上设计了一 种新型复合孤岛检测方法, 该方法具体为通过检测PCC点
电压频率朝某方向的持续变化次数, 从而自适应分段调节 频率偏移正反馈系数, 加速频率偏移, 且若该变化次数超 过 12 次, 频率仍未超过阈值, 则施加 2 个工频周期的负脉 冲电流幅值, 触发欠压保护, 从而检测出孤岛。通过Matlab 
仿真, 此方法能有效地在自动重合闸之前使光伏系统与电 网解列, 对电网谐波影响小，而且孤岛检测盲区小，具有 一定的应用价值。

\section{参考文献}

[1] IEEE recommended practice for utility interface of photovoltaic (PV) System. IEEE Std 929-2000.

[2] Ranade S. J. , Sagi D. R. , Mulpuri R. , et al, Likelihood of Islanding in Distribution Feeders with Photovoltaic Generation [J], in Power Engineering Society General Meeting, 2007:1-6.

[3] Bas V., Kema N. B. V, Probability of islanding in utility networks due to grid connected photovoltaic power systems [J], in Report IEA PVPS T5-07, 2009.

[4] 党克, 杨富磊, 宫译淳, 严干贵. 分布式并网发电系统孤岛检 测方法综述 $[J]$. 电力电子技术, 2016, (01) : 45-48。

[5] 薛士龙, 蒋晨, 耿攀, 李帆.一种改进的带正反馈的主动频移 法仿真 $[J]$. 上海海事大学学报, 2015, (01) : 75-80+85。

[6] 吴云亚, 阚加荣, 谢少军, 汤雨, 薛迎成. 三种频率偏移孤岛 检测方法的性能比较 [J]. 电网技术, 2016, (03) : 964-971。

[7] 杨恢东, 吴浪, 李心茹, 刘辉, 王河深. 改进的周期主动频移 式孤岛检测方法 $[\mathrm{J}]$. 电力系统保护与控 制, 2016, (16): 50-55。

[8] 林明耀, 顾娟, 单竹杰, 张怡然. 一种实用的组合式光伏并网 系统孤岛效应检测方法 $[\mathrm{J}]$. 电力系统自动 化, 2009, (23) : 85-89。

［9］周诗嘉, 陆格文, 黎涛, 罗隆福. 自适应主动频率偏移孤岛检 测新方法 $[\mathrm{J}]$. 电力系统及其自动化学报, 2012，(02) : 78-82。

[10］应展烽, 陈运运, 田亚生, 吴军基, 冯凯. 基于抗干扰六点测 频法的主动频移孤岛检测 $[\mathrm{J}]$. 电力自动化设 备, 2013, (04)：72-76。
[11] 李洋. 主动电流幅值扰动的孤岛检测方法 $[\mathrm{J}]$. 电气电子教 学学报, 2016,(03):67-70+87。

[12] 崇志强, 戴志辉, 焦彦军. 基于负序电流注入的光伏并网逆 变器孤岛检测方法 $[\mathrm{J}]$. 电力系统保护与控 制, 2014, (24):65-71。

[13] Woyte A, Belmans R, Njis J. Testing the islanding protection function of photovoltaic inverters [C], Power Engineering Society General Meeting, 2003.

[14] 吕振, 杨丽. 并网光伏发电系统的孤岛检测 $[J]$, 中国电力, 2009, 42(3), 71-74。

[15] 郑飞. 光伏并网发电系统关键控制技术研究 [D]. 2011年, 东 南大学。

[16] 刘方锐, 康勇, 张宇. 带正反馈的主动移频孤岛检测法的参 数优化 $[J]$, 电工电能新技术, 2008,27 (3)，22-25。

[17] 李秉键, 吴华波. 基于频差正反馈的孤岛效应检测方法 $[\mathrm{J}]$, 电力电子技术， $2011 ， 45(5) ， 1-3$ 。

[18] 刘芙蓉, 康勇, 段善旭等. 主动移频式孤岛检测方法的参数 优化 $[J]$ 。中国电机工程学报, 2008, (01) : 95-99。

[19] 刘传洋, 何礼高. 基于相位突变与电流扰动结合的并网孤岛 检测 $[J]$. 通信电源技术, 2009, (06) : 6-9。

[20］吴铁洲, 熊金龙, 曾艺师, 张琪. 改进的负序电流扰动孤岛检 测方法 $[J]$. 电力电子技术, 2015, (11) : 36-39。

[21] 黎璋霞, 罗晓曙, 廖志贤, 陈亚欢. 改进的正反馈主动频移式 孤岛检测方法 $[\mathrm{J}]$. 电力系统及其自动化学 报, 2015, (04) : 13-17。

[22］陈超波, 李继超, 高嵩, 宋鹤. 一种快速小盲区的主动移频式 孤岛检测方法研究 [J]. 电源学报, 2015, (04) : 93-100。

[23] 蒋翠, 祁新梅, 郑寿森. 带电压频率正反馈的主动移频式孤 岛检测方法 $[J]$. 电力系统保护与控制, 2014, (08) : 95-100。 